\title{
Metrological resolution and minimum uncertainty states in linear and nonlinear signal detection schemes
}

\author{
Daniel Maldonado-Mundo and Alfredo Luis* \\ Departamento de Óptica, Facultad de Ciencias Físicas, Universidad Complutense, 28040 Madrid, Spain
}

(Received 6 September 2009; published 4 December 2009)

\begin{abstract}
We study the performance of linear and nonlinear optical schemes for the detection of weak signals for two classes of probe states. These are quadrature coherent squeezed states and the minimum uncertainty states of the generator of the transformation and the measured observable. Both for linear and nonlinear schemes we show that the generator-measurement minimum uncertainty states are far from being optimum, while the quadrature coherent squeezed states can reach maximum accuracy almost for the same amount of squeezing in both cases. The analysis is largely based on a suitable approximation treating the photon number as a continuous variable.
\end{abstract}

DOI: 10.1103/PhysRevA.80.063811

PACS number(s): 42.50.St, 42.50.Dv, 03.65.-w

\section{INTRODUCTION}

The basic objective of quantum metrology is to infer the unknown value $\chi$ of a given signal as accurately as possible. This is a key practical issue of quantum mechanics involving fundamental concepts such as uncertainty relations, complementarity, and nonclassical properties.

Signals $\chi$ are encoded on quantum states by $\chi$-dependent transformations $U_{\chi}$ acting on an input probe state $|\psi\rangle$, usually of the form $U_{\chi}=\exp (i \chi G)$, where $G$ is the generator of the transformation. The unknown value $\chi$ of the signal is inferred from a measurement $M$ performed on the output probe state $\left|\psi_{\chi}\right\rangle=U_{\chi}|\psi\rangle$. In this work we will focus on probe states in a single mode of the quantum electromagnetic field, although equivalent conclusions can be derived for material systems. The objective of this work is to study the performance of some linear and nonlinear schemes for two classes of probe states: quadrature coherent squeezed states and the minimum uncertainty states of the pair $G, M$.

The performance (or resolution) of the detection process is determined by four basic ingredients: (a) the measurement $M$, (b) the generator $G$, (c) the probe state $|\psi\rangle$, and (d) the data analysis. Let us specify these ingredients for this work:

(a) For definiteness we fix the measurement $M$ to be a field quadrature because this corresponds to simple interferometric schemes.

(b) For the generator $G$ we consider two possibilities: the photon-number operator, representing propagation in optically linear media, and its square, representing propagation in optically nonlinear media. The linear case is standard spectroscopy and interferometry, where the minimum signal uncertainty is given by the Heisenberg limit [1-3]. The interest of the nonlinear case relies on the fact that it can beat the Heisenberg limit [3-5].

(c) We consider two kinds of probes. The first class is the minimum uncertainty states of the pair $G, M$. This choice is suggested by their reduced fluctuations concerning the two only observables involved in the process. The second class is the quadrature coherent squeezed states that have proven

\footnotetext{
*alluis@ fis.ucm.es; http://www.ucm.es/info/gioq/alfredo.html
}

their usefulness in quantum metrology and can be generated experimentally [6].

(d) As performance measures we will consider the signal to noise ratio and some intrinsic resolution measures in terms of the distance between the input $|\psi\rangle$ and the output $\left|\psi_{\chi}\right\rangle$ probe states [7-10].

The main original results of this work are:

(i) We demonstrate that the minimum uncertainty states of the generator-measurement pair are far from being optimum both in the linear and nonlinear cases. This is rather paradoxical since this is often considered as a prerequisite to optimize detection processes.

(ii) Both in the linear and nonlinear cases the quadrature coherent squeezed states can reach the maximum resolution allowed by the theory, almost for the same amount of squeezing in both cases. This is to say that the same squeezed states are approximately optimum for linear and nonlinear schemes simultaneously.

(iii) The above results are confirmed by the different performance estimators employed.

(iv) We derive suitable approximations to obtain practical expressions for the generator-measurement minimum uncertainty states. This is done by considering the photon-number variable as continuous instead of discrete. This is allowed by the large number of photons required to detect weak signals.

(v) Moreover, we approximate the generator-measurement minimum uncertainty states by quadrature coherent squeezed states. The result is consistent with point (iv) and allows us to approximate rather academic states by practical states [11].

In Sec. II we provide the basic framework by recalling the performance measures to be used and developing the approximations mentioned in points (iv) and (v). This is particularized in Sec. III to linear transformations generated by the photon-number operator and in Sec. IV to nonlinear transformations generated by the square photon-number operator.

Finally, we can mention some alternatives to the choices in points (a) and (d) above. Optimal performances are usually obtained with covariant measurements, where the transformation $U_{\chi}$ produces just a translation of the measured observable. For linear generators this is essentially the phase 
[12], while for the nonlinear case this is a slightly more involved observable. In any case such measurements have very difficult practical implementation, so we have rather focused on experimentally feasible phase-dependent observables, such as the field quadratures.

Concerning performance measures we focus on signal to noise ratio for simplicity. The main conclusions are reproduced by more powerful estimators such as the quantum Fisher information. Another meaningful performance measure is provided by the relative entropy, or the KullbackLeibler divergence, which assesses how apart are the statistics corresponding to two different signal values $[13,14]$. In particular this allows us to introduce a global distinguishability depending exclusively on the probability distributions by integrating the relative entropy for signal values within a given interval determined a priori [14]. This is in contrast with assuming a definite value for the unknown signal that leads to local estimation performances (such as the Fisher information, for example) depending on derivatives of the probability distributions. Local and global measures may provide slightly different types of physical information $[14,15]$.

\section{FORMULATION AND BASIC RELATIONS}

Throughout we will focus on a single mode of the electromagnetic field with complex amplitude operator $a$ satisfying the usual bosonic commutation relations $\left[a, a^{\dagger}\right]=1$. The real and imaginary parts of the complex amplitude $a=(X$ $+i Y) / 2$ are the field quadratures

$$
X=a+a^{\dagger}, \quad Y=i\left(a^{\dagger}-a\right)
$$

that satisfy the commutation relation $[X, Y]=2 i$, being equivalent to position and linear momentum. Besides quantum optics, the analysis and results below can be equally well applied to material systems such as trapped ions.

\section{A. Generators and measurement}

We will focus on transformations generated by powers of the photon-number operator $N=a^{\dagger} a$, in particular $G=N$ and $G=N^{2}$. The case $G=N$ describes light propagation in linear media (this is standard interferometry), while the case $G$ $=N^{2}$ describes propagation in nonlinear media.

Throughout we will consider the measurement of the quadrature $Y$ (equivalent to linear momentum for material systems) that can be easily implemented in interferometric schemes. This is because for large photon numbers (always the case in precision signal detection) intensity measurements at the output of a two-beam interferometer can be suitably described as a single-mode quadrature measurement by approximating the bright mode containing most of the photons by a classical field amplitude [16].

\section{B. Resolution}

We will consider two estimators of the performance of the signal detection process. In both cases the signal is assumed to be very weak $\chi \ll 1$ since we are interested in very precise measurements limited by quantum noise.

\section{Noise to signal ratio}

The most simple and widely used performance measure is the noise to signal ratio. The variation of the measured observable caused by weak signals is

$$
Y(\chi)=\exp (-i \chi G) Y \exp (i \chi G) \simeq Y+i \chi[Y, G] .
$$

Following the unit signal to noise ratio criterion, the signal $\Delta \chi$ that produces a shift of the outcomes $|Y(\Delta \chi)-Y(0)|$ that equal the background noise $\Delta Y$ is

$$
\Delta \chi \simeq \frac{\Delta Y}{|\langle[Y, G]\rangle|} \geq \frac{1}{2 \Delta G},
$$

where $\Delta Y, \Delta G$, and $\langle[Y, G]\rangle$ are evaluated in the input probe state $|\psi\rangle$, and for the last inequality the generatormeasurement uncertainty relation has been used,

$$
\Delta Y \Delta G \geq \frac{1}{2}|\langle[Y, G]\rangle| .
$$

Equation (2.3) suggests that a suitable strategy to minimize $\Delta \chi$ is to focus on the minimum uncertainty states satisfying Eqs. (2.3) and (2.4) as equalities.

For $G=N^{k}$ it has been shown that the minimum $\Delta \chi$ is a function of the mean number of photons $\langle N\rangle$ that scales as $[2,3]$

$$
\left.\Delta \chi\right|_{\min } \propto \frac{1}{\langle N\rangle^{k}} .
$$

For linear transformations $k=1$ this is the Heisenberg limit, so that the signal uncertainty is inversely proportional to the mean number of photons of the probe state [1-3]. For nonlinear transformations $k \geq 2$ it has been shown that the Heisenberg limit can be surpassed even with semiclassical probe states $[4,5]$.

\section{Intrinsic resolution measures}

There are other performance measures that are intrinsic in the sense that they do not depend on the measurement $M$ performed on the transformed probe state. They are based on the distance between the input $\rho$ and output $\rho_{\chi}$ density matrices of the probe. This is the case of the Bures distance, leading to the quantum Fisher information $F$ as $\chi \rightarrow 0[7,10]$,

$$
D_{B}^{2}\left(\rho, \rho_{\chi}\right)=2\left(1-\operatorname{tr} \sqrt{\sqrt{\rho} \rho_{\chi}} \sqrt{\rho}\right) \simeq \chi^{2} F,
$$

where

$$
F=\frac{1}{2} \sum_{k, \ell} \frac{\left(p_{k}-p_{\ell}\right)^{2}}{p_{k}+p_{\ell}}\left|\left\langle\psi_{k}|G| \psi_{\ell}\right\rangle\right|^{2},
$$

and $\left|\psi_{k}\right\rangle, p_{k}$ are the eigenvectors and eigenvalues of $\rho$, respectively, including the vanishing eigenvalues. Simpler expressions are obtained using the Hilbert-Schmidt distance leading to the function $\Lambda$ as $\chi \rightarrow 0$ [7-9],

$$
D_{H S}^{2}\left(\rho, \rho_{\chi}\right)=\operatorname{tr}\left[\left(\rho-\rho_{\chi}\right)^{2}\right] \simeq 2 \chi^{2} \Lambda,
$$

where 


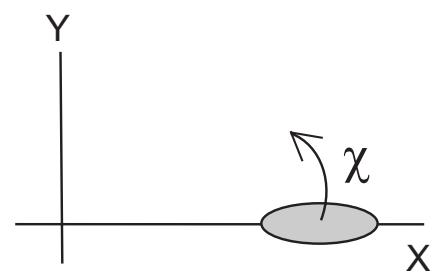

FIG. 1. Sketch illustrating that $\langle Y\rangle=0$ provides maximum variation of $\langle Y\rangle$ for weak signals.

$$
\Lambda=\frac{1}{2} \sum_{j, k}\left(p_{j}-p_{k}\right)^{2}\left|\left\langle\psi_{k}|G| \psi_{j}\right\rangle\right|^{2} .
$$

For pure probe states $\rho=|\psi\rangle\langle\psi|$, which will be the case throughout this work, both $F$ and $\Lambda$ become the variance of the generator $G$ on the input probe state $|\psi\rangle$,

$$
F=\Lambda=(\Delta G)^{2} .
$$

Better performance means larger distances $D_{B}$ and $D_{H S}$, which is equivalent to larger variance $\Delta G$. This is similar to the signal to noise criterion (2.3) but in this case there is no requirement about the minimum uncertainty of the generatormeasurement pair.

\section{Generator-measurement minimum uncertainty states}

The minimum uncertainty states of the generatormeasurement uncertainty relation (2.4) are given by the solution of the eigenvalue equation

$$
\left(N^{k}+i \lambda Y\right)|\psi\rangle=\mu|\psi\rangle,
$$

where $\lambda$ and $\mu$ are real with

$$
\left\langle N^{k}\right\rangle=\mu, \quad\langle Y\rangle=0, \quad \lambda=\frac{\Delta N^{k}}{\Delta Y},
$$

so that the criterion of unit noise to signal ratio becomes

$$
\Delta \chi=\frac{1}{2 \Delta N^{k}},
$$

where $\Delta N^{k}=\sqrt{\left\langle N^{2 k}\right\rangle-\left\langle N^{k}\right\rangle^{2}}$. We have considered $\langle Y\rangle=0$ since in such a case the variation of $\langle Y\rangle$ caused by weak signals will be maximum for fixed $\langle N\rangle$, as illustrated in Fig. 1.

Expressing $|\psi\rangle$ in the photon-number basis

$$
|\psi\rangle=\sum_{n=0}^{\infty} \psi(n)|n\rangle
$$

with $N|n\rangle=n|n\rangle$, we get

$$
n^{k} \psi(n)+\lambda[\sqrt{n+1} \psi(n+1)-\sqrt{n} \psi(n-1)]=\mu \psi(n),
$$

leading to the recurrence relation

$$
\psi(n+1)=\frac{\mu-n^{k}}{\lambda \sqrt{n+1}} \psi(n)+\sqrt{\frac{n}{n+1}} \psi(n-1) .
$$

This can be solved exactly for $k=1$ [17] (see below), but no solutions are known for $k>1$. Thus, to address the nonlinear case $k>1$ we consider the following approximations.

\section{Approximation by continuous number}

In order to solve Eq. (2.15) let us consider an approximation where the number $n$ is treated as a continuous variable. This is justified when $\psi(n)$ takes significant values only for $n \gg 1$, which always holds for precise detection, and provided that $\psi(n)$ depends slowly on $n$, so that we may write

$$
\psi(n \pm 1) \simeq \psi(n) \pm \frac{d}{d n} \psi(n) .
$$

Using $\sqrt{n+1} \simeq \sqrt{n}$ in Eq. (2.15) we get

$$
n^{k} \psi(n)+\lambda \sqrt{n}[\psi(n+1)-\psi(n-1)]=\mu \psi(n),
$$

which using Eq. (2.17) leads to

$$
n^{k} \psi(n)+2 \lambda \sqrt{n} \frac{d}{d n} \psi(n)=\mu \psi(n),
$$

which can be solved in the form

$$
\psi(n) \propto \exp \left\{-\frac{\sqrt{n}}{(2 k+1) \lambda}\left[n^{k}-(2 k+1) \mu\right]\right\} .
$$

Let us note that in the continuous-number approximation (2.17) the quadrature operators $X, Y$ become

$$
Y=i\left(a^{\dagger}-a\right) \simeq-2 i \sqrt{n} \frac{d}{d n}, \quad X=a^{\dagger}+a \simeq 2 \sqrt{n},
$$

which satisfy the same commutation relations of their exact counterparts,

$$
[X, Y]=\left[2 \sqrt{n},-2 i \sqrt{n} \frac{d}{d n}\right]=2 i .
$$

Concerning the generator-measurement commutator representation (2.21) leads to

$$
\left[Y, N^{k}\right] \simeq-2 i k N^{k-1 / 2},
$$

so that the generator-measurement uncertainty relation is of the form

$$
\Delta Y \Delta N^{k} \geq k\left\langle N^{k-1 / 2}\right\rangle
$$

\section{Gaussian approximation}

Solution (2.20) is still of limited use because of the difficulty of integration. It can be further simplified if we approximate $\psi(n) \propto \exp [-f(n)]$ in Eq. (2.20) by a Gaussian in the form

$$
f(n) \simeq f(\bar{n})+\left.\frac{1}{2} \frac{d^{2} f(n)}{d n^{2}}\right|_{n=\bar{n}}(n-\bar{n})^{2},
$$

where $\bar{n}$ is given by the condition 


$$
\left.\frac{d f(n)}{d n}\right|_{n=\bar{n}}=0,
$$

leading to

$$
\psi(n) \simeq \frac{1}{\left[2 \pi(\Delta N)^{2}\right]^{1 / 4}} \exp \left[-\frac{(n-\langle N\rangle)^{2}}{4(\Delta N)^{2}}\right],
$$

where

$$
\langle N\rangle \simeq \mu^{1 / k}, \quad(\Delta N)^{2} \simeq \frac{\lambda}{k} \mu^{(3-2 k) /(2 k)} .
$$

We will see below that these approximations fit quite well with the exact $\psi(n)$.

From Eq. (2.12) we have $\left\langle N^{k}\right\rangle=\mu$ while after Eq. (2.28) we get $\langle N\rangle^{k}=\mu$. This means that $\left\langle N^{k}\right\rangle \simeq\langle N\rangle^{k}$ which for $k$ $>1$ implies $\Delta N \ll\langle N\rangle$; and, after Eq. (2.28),

$$
\lambda \ll k\langle N\rangle^{k+1 / 2} \text {. }
$$

This allows us to simplify some moments of the number operator $\left\langle N^{\ell}\right\rangle$ to be used below by retaining just the leading terms in $\langle N\rangle$. More specifically, since $\left\langle N^{\ell}\right\rangle=\left\langle(\langle N\rangle+\delta)^{\ell}\right\rangle$, with $\delta=N-\langle N\rangle$, and taking into account $|\delta| \ll\langle N\rangle$ we get retaining just the two first leading terms in $\langle N\rangle$,

$$
\left\langle N^{\ell}\right\rangle \simeq\langle N\rangle^{\ell}+\frac{1}{2} \ell(\ell-1)\langle N\rangle^{\ell-2}(\Delta N)^{2},
$$

so that

$$
\left(\Delta N^{k}\right)^{2} \simeq k^{2}\langle N\rangle^{2 k-2}(\Delta N)^{2} .
$$

Concerning the $X, Y$ uncertainties a direct computation using the operator approximations (2.21) acting on the Gaussian wave function (2.27) leads to

$$
(\Delta Y)^{2} \simeq \frac{1}{(\Delta X)^{2}} \simeq \frac{k}{\lambda} \mu^{(2 k-1) /(2 k)},
$$

where we have used that $\Delta N \ll\langle N\rangle$.

It can be checked that the approximate form (2.24) of the generator-measurement uncertainty relation is satisfied as an equality after approximating $\left\langle N^{k-1 / 2}\right\rangle$ by $\langle N\rangle^{k-1 / 2}$. Moreover, from Eq. (2.32) we get that the generator-measurement minimum uncertainty states are also approximately minimum uncertainty states of the quadratures since $\Delta X \Delta Y \simeq 1$ after Eq. (2.32). This is further analyzed in the next subsection.

\section{Linear approximation of the eigenvalue equation}

Alternatively, we can approximate directly the eigenvalue equation (2.11) taking into account that for large photon numbers $\mu$ is also large. In such a case we can define the complex amplitude $b$ by the relation

$$
a=b+\sqrt{\mu^{1 / k}},
$$

which when substituted in the eigenvalue equation (2.11) leads to

$$
\left[\left(b^{\dagger} b+\sqrt{\mu^{1 / k}} X_{b}+\mu^{1 / k}\right)^{k}+i \lambda Y_{b}\right]|\xi\rangle=\mu|\xi\rangle,
$$

where $X_{b}, Y_{b}$ are the corresponding quadratures of the complex amplitude $b$, and the state $|\xi\rangle$ is defined in terms of $|\psi\rangle$ via the following unitary transformation:

$$
|\xi\rangle=D\left(\sqrt{\mu^{1 / k}}\right)|\psi\rangle,
$$

where $D(\alpha)=\exp \left(\alpha a^{\dagger}-\alpha^{*} a\right)$ is the displacement operator $D^{\dagger}(\alpha) a D(\alpha)=a+\alpha$. Retaining just the leading terms in $\mu$ in Eq. (2.34) we get the following eigenvalue equation:

$$
\left[k \mu^{(2 k-1) /(2 k)} X_{b}+i \lambda Y_{b}\right]|\xi\rangle=0 .
$$

This means that $|\xi\rangle$ are squeezed vacuum states with

$$
\begin{aligned}
& (\Delta X)^{2}=\left(\Delta X_{b}\right)^{2}=\frac{\lambda}{k} \mu^{(1-2 k) /(2 k),} \\
& (\Delta Y)^{2}=\left(\Delta Y_{b}\right)^{2}=\frac{k}{\lambda} \mu^{(2 k-1) /(2 k)} .
\end{aligned}
$$

This coincides with Eq. (2.32), so that the continuousnumber and Gaussian approximations are equivalent to this linear approximation of the eigenvalue equation. Therefore, for large photon numbers the generator-measurement minimum uncertainty states can be suitably approximated by a quadrature coherent squeezed state.

\section{LINEAR TRANSFORMATIONS}

Let us particularize the above general expressions to linear transformations with generator $G=N$. This corresponds to light propagation in linear media, leading to linear inputoutput relations between complex amplitudes.

\section{A. Generator-measurement minimum uncertainty states}

\section{Exact solution}

For $G=N$ there is an exact solution for the generatormeasurement minimum uncertainty states that in the photonnumber basis reads [17]

$$
\psi(n)=\left(\frac{m !}{n !}\right)^{[\operatorname{sgn}(n-m)] / 2} \frac{\exp \left(-\lambda^{2} / 2\right)}{\left[L_{m}^{0}\left(-4 \lambda^{2}\right)\right]^{1 / 2}} \lambda^{|n-m|} L_{m}^{|n-m|}\left(-\lambda^{2}\right),
$$

where $L_{m}^{\ell}$ are the Laguerre polynomials, $\operatorname{sgn}(x)$ is the sign of $x$, and $m$ is a natural number so that

$$
\mu=\langle N\rangle=m+\lambda^{2} .
$$

The exact number distribution $|\psi(n)|^{2}$ is plotted as squares in Fig. 2 for $m=5$ and $\mu=100$.

A key point of this solution is that, since $m \geq 0$, we have

$$
\lambda \leq \sqrt{\langle N\rangle} .
$$

For the minimum $\lambda=0$ the solution is the number state $|\psi\rangle$ $=|m\rangle$ with $\Delta N=0$, while for the maximum $\lambda=\sqrt{\langle N\rangle}$ the solution is a quadrature coherent state $|\alpha\rangle$ with complex amplitude $\alpha=\sqrt{\langle N\rangle}$ and $\Delta N=\sqrt{\langle N\rangle}$.

Focusing on the signal resolution, in Fig. 3 we have plotted $\Delta N$ as a function of $m$ for $\mu=20$. The maximum number uncertainty $\Delta N=\sqrt{\langle N\rangle}$ occurs for $m=0$ and $\lambda=\sqrt{\langle N\rangle}$ so that the minimum $\Delta \chi$ in Eq. (2.13) scales as 


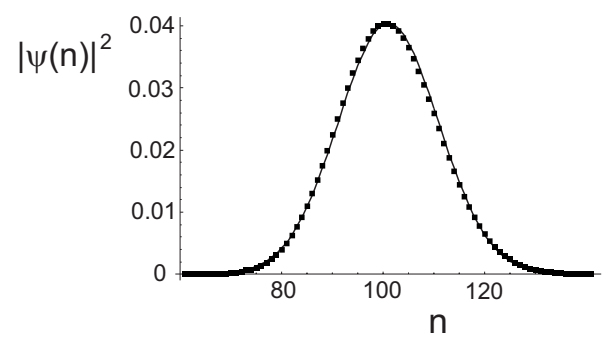

FIG. 2. Plot of the photon-number distribution $|\psi(n)|^{2}$ as a function of $n$ for the generator-measurement minimum uncertainty states with $m=5$ and $\mu=100$. The squares are the exact values while the solid line is the result of the continuous-number and Gaussian approximations.

$$
\Delta \chi=\frac{1}{2 \sqrt{\langle N\rangle}} \gg \frac{1}{\langle N\rangle},
$$

where the inequality holds for $\langle N\rangle \gg 1$ (always the case in precision measurements). This is that the signal uncertainty provided by the generator-measurement minimum uncertainty states is very far from the absolute theoretical minimum (2.5) for $k=1$ (Heisenberg limit).

From Eq. (2.32) we have $(\Delta Y)^{2} \simeq \sqrt{\langle N\rangle} / \lambda$ and because of the upper bound (3.3) we have always $\Delta Y \geq 1$. This means that these states never present reduced quantum fluctuations in the quadrature $Y$. This prevents their metrological usefulness in this scheme since this requires squeezing in the $Y$ quadrature as illustrated in Fig. 1. In other words, they are amplitude squeezed states while precision detection requires phase squeezed states.

\section{Approximate solution}

The continuous-number approximation for $k=1$ in Eq. (2.20) gives

$$
\psi(n) \propto \exp \left\{-\frac{\sqrt{n}}{3 \lambda}(n-3 \mu)\right\},
$$

which when approximated by a Gaussian gives Eq. (2.27) with, after Eqs. (2.28) and (2.32),

$$
\langle N\rangle=\mu, \quad(\Delta N)^{2}=\lambda \sqrt{\langle N\rangle},
$$

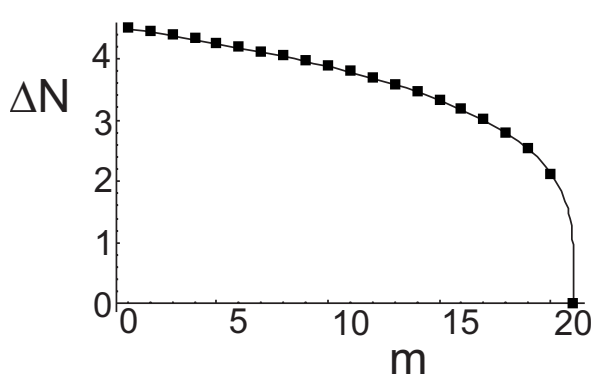

FIG. 3. Plot of $\Delta N$ as a function of $m$ for generatormeasurement minimum uncertainty states for $\mu=20$. The squares are the exact values while the solid line is the result of the continuous-number and Gaussian approximations.

$$
(\Delta Y)^{2}=\frac{1}{(\Delta X)^{2}}=\frac{\sqrt{\langle N\rangle}}{\lambda} .
$$

The Gaussian photon-number distribution is plotted as solid line in Fig. 2 for $\mu=100$ and $m=5$. The Gaussian and the distribution (3.5) are indistinguishable. It can be appreciated in Fig. 2 that these approximations are very close to the exact results for the range of parameters of interest; this is large $\langle N\rangle$ and $\lambda$. Figure 3 shows that the approximations give very accurate results for $\Delta N$.

\section{B. Optimum quadrature coherent squeezed states}

Next we examine the resolution that can be achieved in linear schemes with quadrature coherent squeezed states as probe states. This is given by

$$
\Delta \chi \simeq \frac{\Delta Y}{|\langle[Y, N]\rangle|}=\frac{\Delta Y}{|\langle X\rangle|} .
$$

Let us express the right-hand side of the equation in terms of $\langle N\rangle$ by using the relation

$$
N=\frac{1}{4}\left(X^{2}+Y^{2}-2\right) \simeq \frac{1}{4} X^{2},
$$

where we have taken into account that the $Y$ contribution is negligible since we are always interested in the case $\langle Y\rangle=0$ and $\Delta Y \ll 1$. Then we have

$$
\langle N\rangle \simeq \frac{1}{4}\left\langle X^{2}\right\rangle=\frac{1}{4}\left(\langle X\rangle^{2}+\eta\right),
$$

where $\eta=(\Delta X)^{2}=1 /(\Delta Y)^{2}$. Therefore,

$$
\Delta \chi \simeq \frac{1}{\sqrt{\eta(4\langle N\rangle-\eta)}} \geq \frac{1}{2\langle N\rangle},
$$

where the equality (i.e., the minimum $\Delta \chi$ ) is reached for $\eta$ $=2\langle N\rangle$ so that $\langle X\rangle=\Delta X=\sqrt{2\langle N\rangle}$. This is to say that the quadrature coherent squeezed states can reach the maximum resolution in Eq. (2.5) for $k=1$ (Heisenberg limit).

Furthermore, for $\eta=2\langle N\rangle$,

$$
(\Delta Y)^{2}=\frac{1}{2\langle N\rangle} \ll 1 .
$$

This means that, unlike the generator-measurement minimum uncertainty states, these optimum states are squeezed in the appropriate direction to improve the accuracy (phase squeezed states), as illustrated in Fig. 1.

\section{Intrinsic performance measures}

The above conclusions can be derived also from the intrinsic performance measures (2.6) and (2.8). Since we are considering probes in pure states, the maximum intrinsic resolution is given by the maximum $\Delta N$. For the generatormeasurement minimum uncertainty states the maximum $\Delta N$ is

$$
(\Delta N)^{2}=\langle N\rangle .
$$

For the optimum quadrature coherent squeezed states we can compute $\Delta N$ using again approximation (3.9) and taking 
into account that the $X$ distribution is Gaussian with $\langle X\rangle$ $=\Delta X=\sqrt{2\langle N\rangle}$. Thus, we get

$$
(\Delta N)^{2}=1.5\langle N\rangle^{2} .
$$

The key point is that $\Delta N$ for the optimum quadrature coherent squeezed states is much larger than the maximum $\Delta N$ in Eq. (3.13) for generator-measurement minimum uncertainty states. Thus, the intrinsic performance measures confirm the results of the above analysis.

\section{Imperfections and large signals}

This work focuses on the unavoidable performance limits caused by quantum physics, so that we have assumed detection schemes free from technical imperfections that in principle might be avoided or reduced to tolerable levels. Also, we have assumed small signals. Next we discuss the consequences on the detection performance of removing these assumptions.

\section{Imperfections}

Losses, finite quantum efficiency, and other decoherence processes affect the detection performance, especially if the probe state is strongly nonclassical, as it is usually the case for optimum probe states [18]. For the sake of simplicity, to discuss this point we mainly focus on the signal to noise ratio criterion in the linear scheme.

Maybe the simplest description of losses and nonideal detectors is achieved by inserting a fictitious beam splitter of transmittance $t \leq 1$ in an otherwise ideal scheme, mixing the output probe state $\rho_{\chi}$ with vacuum. This is equivalent to saying that the measured observable is $\tilde{Y}=\sqrt{t} Y+\sqrt{1-t} Y_{0}$, where $Y_{0}$ is a field quadrature of the vacuum mode with $\Delta Y_{0}=1$. The signal resolution (3.8) becomes

$$
(\Delta \chi)^{2} \simeq \frac{(\Delta \tilde{Y})^{2}}{|\langle[\tilde{Y}, G]\rangle|^{2}} \simeq \frac{t(\Delta Y)^{2}+1-t}{t\langle X\rangle^{2}} .
$$

This has no critical consequences for probes in a coherent state since in such a case $\Delta Y=1$ and Eq. (3.15) merely implies the reduction by a factor $t$ of the effective number of photons. However, for probes in quadrature squeezed states $\Delta Y \ll 1$ and the resolution is heavily spoiled for small $t$ to the extent that quadrature squeezing becomes useless and the best strategy becomes to use coherent states. This is confirmed by the Fisher information [5]. Moreover, this highlights a key property of nonlinear schemes since they allow a robust surpassing of the Heisenberg limit with coherent states $[4,5]$.

\section{Large signals}

Concerning the effect of signal values $\chi$ beyond $\chi \simeq 0$, we get that in such a case the signal to noise ratio becomes

$$
(\Delta \chi)^{2}=\frac{\left(\Delta Y_{\chi}\right)^{2}}{\left\langle X_{\chi}\right\rangle^{2}}=\frac{(\Delta Y)^{2}}{\langle X\rangle^{2}}+\tan ^{2} \chi \frac{(\Delta X)^{2}}{\langle X\rangle^{2}},
$$

where $X_{\chi}$ and $Y_{\chi}$ are the rotated quadrature operators related to the original ones $X$ and $Y$ by the finite phase shift $\exp \left(i \chi a^{\dagger} a\right)$,

$$
Y_{\chi}=\cos \chi Y+\sin \chi X, \quad X_{\chi}=\cos \chi X-\sin \chi Y,
$$

and we have taken into account that for the optimal situation schematized in Fig. $1\langle Y\rangle=0$ and $\langle(X Y+Y X)\rangle=0$. We get that the signal uncertainty increases in a paraboliclike way around its minimum at $\chi=0$. The increase is proportional to the fluctuations $\Delta X$ of the quadrature $X$ conjugate to the measured one, so that $\Delta X$ increases when $\Delta Y$ decreases. Nevertheless, small deviations of $\chi$ around the optimum $\chi=0$ do not lead to dramatic changes in the resolution [19]. Concerning the intrinsic criteria we have that both $F\left(\rho_{\chi}\right)$ and $\Lambda\left(\rho_{\chi}\right)$ are actually independent of $\chi[9,19,20]$. This is clear in Eqs. (2.7) and (2.9) since $\left[U_{\chi}, G\right]=0$.

\section{NONLINEAR TRANSFORMATIONS}

Next we consider nonlinear input-output transformations generated by $G=N^{2}$. In optics this corresponds to the propagation through nonlinear media producing nonlinear inputoutput relations between complex amplitudes. They are interesting since they allow us to beat the Heisenberg limit [3-5]. There is no known analytical solution of the eigenvalue equation (2.11) so we focus directly on the approximate solutions.

\section{A. Generator-measurement minimum uncertainty states: Approximate solution}

The case $k=2$ of Eq. (2.20) gives

$$
\psi(n) \propto \exp \left\{-\frac{\sqrt{n}}{5 \lambda}\left(n^{2}-5 \mu\right)\right\},
$$

which can be further approximated by the Gaussian (2.27) with, after Eqs. (2.28) and (2.32),

$$
\begin{gathered}
\langle N\rangle=\sqrt{\mu}, \quad(\Delta N)^{2}=\frac{\lambda}{2 \sqrt{\langle N\rangle},} \\
(\Delta Y)^{2}=\frac{1}{(\Delta X)^{2}}=\frac{2}{\lambda}\langle N\rangle^{3 / 2} .
\end{gathered}
$$

In Fig. 4 we have plotted the photon-number distribution $|\psi(n)|^{2}$ as a function of $n$ for $\mu=9 \times 10^{3}$ and $\lambda=2 \times 10^{3}$. The squares are the result of the numerical computation of the exact recurrence relation (2.16), while the solid line represents both the continuous-number and Gaussian approximations (they are indistinguishable).

As in the linear case, the existence of an upper bound for $\lambda$ is crucial in this context. In the nonlinear case $k=2$, within continuous-number and Gaussian approximations, we have from Eq. (2.29) that

$$
\lambda \ll 2\langle N\rangle^{5 / 2} .
$$

Concerning the metrological resolution, after Eqs. (2.31), (4.2), and (4.4), the variance of the generator is

$$
\left(\Delta N^{2}\right)^{2} \simeq 4\langle N\rangle^{2}(\Delta N)^{2} \ll\langle N\rangle^{4},
$$

which implies that the signal uncertainty is far above the theoretical minimum in Eq. (2.5) for $k=2$, 


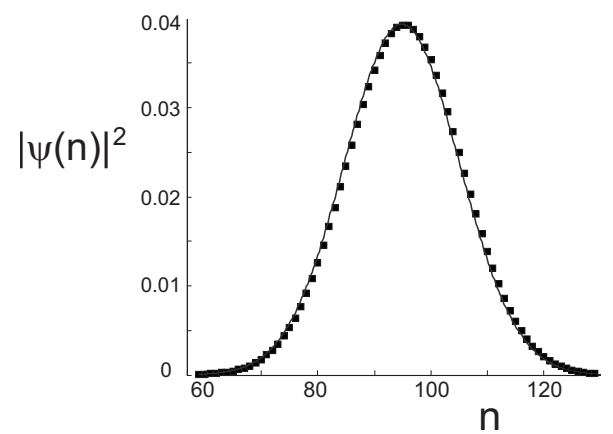

FIG. 4. Plot of the photon-number distribution $|\psi(n)|^{2}$ as a function of $n$ for the generator-measurement minimum uncertainty states with $\mu=9 \times 10^{3}$ and $\lambda=2 \times 10^{3}$. The squares are the result of the numerical evaluation of the recurrence relation (2.16) while the solid line is the result of the continuous-number and Gaussian approximations.

$$
\Delta \chi \simeq \frac{1}{2 \Delta N^{2}} \gg \frac{1}{\langle N\rangle^{2}} .
$$

\section{B. Optimum quadrature squeezed states}

Next we examine the resolution that can be achieved when the probe is prepared in a quadrature coherent squeezed state,

$$
\Delta \chi \simeq \frac{\Delta Y}{\left|\left\langle\left[Y, N^{2}\right]\right\rangle\right|}=\frac{\Delta Y}{\langle(N X+X N)\rangle} .
$$

Taking into account that the $Y$ contribution is negligible since $\langle Y\rangle=0$ and $\Delta Y \ll 1$ we can evaluate the denominator using approximation (3.9) and the Gaussian character of the $X$ distribution,

$$
\langle(N X+X N)\rangle \simeq \frac{1}{2}\left\langle X^{3}\right\rangle=\frac{1}{2}\left(\langle X\rangle^{3}+3 \eta\langle X\rangle\right),
$$

where as before $\eta=(\Delta X)^{2}=1 /(\Delta Y)^{2}$. From Eq. (3.10) we have $\langle X\rangle=\sqrt{4\langle N\rangle-\eta}$ so we can express $\Delta \chi$ as a function of $\langle N\rangle$ and $\eta$ and then find its minimum for fixed $\langle N\rangle$ when $\eta$ is varied. This leads to

$$
\Delta \chi \geq \frac{0.1}{\langle N\rangle^{2}},
$$

where the equality is reached for $\eta=(1+\sqrt{3})\langle N\rangle=2.7\langle N\rangle$ and

$$
(\Delta Y)^{2} \propto \frac{0.4}{\langle N\rangle} .
$$

The conclusion is that the quadrature coherent squeezed states can reach the maximum resolution allowed with the nonlinear generator $G=N^{2}$ and quadrature detection. Comparing Eqs. (3.12) and (4.10) we can see that this is achieved by essentially the same amount of quadrature squeezing required to reach the maximum resolution allowed by linear transformations (Heisenberg limit). This is interesting since the minimum uncertainty (4.9) is far below the Heisenberg limit.

\section{Intrinsic performance measures}

The above conclusions are again consistent with the intrinsic performance measures (2.6) and (2.8). This is because for the generator-measurement minimum uncertainty states we have the upper bound $\left(\Delta N^{2}\right)^{2} \ll\langle N\rangle^{4}$ in Eq. (4.5) for the uncertainty of the generator. On the other hand, to compute $\Delta N^{2}$ for the optimum quadrature coherent squeezed states we can use again approximation (3.9). Taking into account that the $X$ distribution is Gaussian with $\langle X\rangle \simeq 1.1 \sqrt{\langle N\rangle}$ and $\Delta X$ $\simeq 1.6 \sqrt{\langle N\rangle}$, we get

$$
\left(\Delta N^{2}\right)^{2} \simeq 53\langle N\rangle^{4} .
$$

The uncertainty $\Delta N^{2}$ in Eq. (4.11) for the optimum quadrature coherent squeezed states is much larger than $\Delta N^{2}$ for the generator-measurement minimum uncertainty states in Eq. (4.5). This implies the same relation for the corresponding distances $D_{H S}$ and $D_{B}$, in agreement with the results of the above analysis.

\section{CONCLUSIONS}

We have shown that the minimum uncertainty states of the generator-measurement pair are far from being optimum both in the cases of linear and nonlinear transformations. This is rather paradoxical since this is often considered as a prerequisite to optimize detection processes according to the common unit signal to noise ratio criterion.

Both in the linear and nonlinear cases the quadrature coherent squeezed states can reach the maximum resolution allowed by the theory. It is worth noting that essentially the same amount of squeezing is required in both cases. These conclusions have been confirmed by different performance estimators.

We have derived these results by developing a suitable approximation considering the photon-number variable as continuous instead of discrete, which is allowed by the large number of photons required to detect weak signals. Moreover, we have obtained suitable approximations for the generator-measurement minimum uncertainty states in terms of quadrature coherent squeezed states. The result is consistent with the continuous-number approximation and allows us to approximate rather academic states by practical states.

\section{ACKNOWLEDGMENT}

A.L. acknowledges support from Project No. FIS200801267 of the Spanish Dirección General de Investigación del Ministerio de Ciencia e Innovación. 
[1] B. Yurke, S. L. McCall, and J. R. Klauder, Phys. Rev. A 33, 4033 (1986); D. J. Wineland, J. J. Bollinger, W. M. Itano, and D. J. Heinzen, ibid. 50, 67 (1994); C. Brif and A. Mann, ibid. 54, 4505 (1996); Z. Y. Ou, ibid. 55, 2598 (1997); H. Uys and P. Meystre, ibid. 76, 013804 (2007); U. Dorner, R. Demkowicz-Dobrzanski, B. J. Smith, J. S. Lundeen, W. Wasilewski, K. Banaszek, and I. A. Walmsley, Phys. Rev. Lett. 102, 040403 (2009).

[2] V. Giovannetti, S. Lloyd, and L. Maccone, Phys. Rev. Lett. 96, 010401 (2006).

[3] A. Luis, Phys. Rev. A 76, 035801 (2007).

[4] A. Luis, Phys. Lett. A 329, 8 (2004); S. Boixo, S. T. Flammia, C. M. Caves, and J. M. Geremia, Phys. Rev. Lett. 98, 090401 (2007); S. M. Roy and S. L. Braunstein, ibid. 100, 220501 (2008); S. Boixo, A. Datta, S. T. Flammia, A. Shaji, E. Bagan, and C. M. Caves, Phys. Rev. A 77, 012317 (2008).

[5] J. Beltrán and A. Luis, Phys. Rev. A 72, 045801 (2005).

[6] C. M. Caves, Phys. Rev. D 23, 1693 (1981); M. Xiao, L.-A. Wu, and H. J. Kimble, Phys. Rev. Lett. 59, 278 (1987); P. Grangier, R. E. Slusher, B. Yurke, and A. LaPorta, ibid. 59, 2153 (1987); E. S. Polzik, J. Carri, and H. J. Kimble, ibid. 68, 3020 (1992); A. Luis and L. L. Sánchez-Soto, Opt. Commun. 89, 140 (1992); A. F. Pace, M. J. Collett, and D. F. Walls, Phys. Rev. A 47, 3173 (1993); J. L. Sørensen, J. Hald, and E. S. Polzik, Phys. Rev. Lett. 80, 3487 (1998); H.-A. Bachor, A Guide to Experiments in Quantum Optics (Wiley-VCH, Weinheim, 1998); C. Brif, Phys. Lett. A 263, 15 (1999); N. Treps, U. Andersen, B. Buchler, P. K. Lam, A. Maître, H.-A. Bachor, and C. Fabre, Phys. Rev. Lett. 88, 203601 (2002).

[7] S. Luo and Q. Zhang, Phys. Rev. A 69, 032106 (2004).
[8] V. V. Dodonov, O. V. Man'ko, V. I. Man'ko, and A. Wünsche, J. Mod. Opt. 47, 633 (2000); S. Luo, Phys. Rev. Lett. 91 180403 (2003); V. V. Dodonov and M. B. Renó, Phys. Lett. A 308, 249 (2003); J. Lee, M. S. Kim, and Č. Brukner, Phys. Rev. Lett. 91, 087902 (2003); M. Hendrych, M. Dušek, R. Filip, and J. Fiurášek, Phys. Lett. A 310, 95 (2003).

[9] A. Rivas and A. Luis, Phys. Rev. A 77, 063813 (2008).

[10] S. L. Braunstein and C. M. Caves, Phys. Rev. Lett. 72, 3439 (1994); V. Vedral and M. B. Plenio, Phys. Rev. A 57, 1619 (1998).

[11] A. Aragão, P. B. Monteiro, A. T. Avelar, and B. Baseia, Phys. Lett. A 337, 296 (2005); M. Freyberger and W. Schleich, Phys. Rev. A 49, 5056 (1994); A. Luis, Phys. Lett. A 354, 71 (2006).

[12] G. M. D’Ariano, C. Macchiavello, and M. F. Sacchi, Phys Lett. A 248, 103 (1998); P. Lahti and J.-P. Pellonpää, Phys. Scr. 66, 66 (2002).

[13] T. M. Cover and J. A. Thomas, Elements of Information Theory (Wiley, New York, 1991).

[14] G. A. Durkin and J. P. Dowling, Phys. Rev. Lett. 99, 070801 (2007).

[15] F. Pennini and A. Plastino, Phys. Lett. A 365, 263 (2007).

[16] A. Luis, Opt. Commun. 273, 173 (2007).

[17] Z. Hradil, Phys. Rev. A 44, 792 (1991); A. Lukš, V. Peřinová, and J. Křepelka, J. Mod. Opt. 41, 2325 (1994).

[18] R. Demkowicz-Dobrzanski, U. Dorner, B. J. Smith, J. S. Lundeen, W. Wasilewski, K. Banaszek, and I. A. Walmsley, Phys. Rev. A 80, 013825 (2009).

[19] L. Pezzé and A. Smerzi, Phys. Rev. Lett. 100, 073601 (2008).

[20] S. Luo, Proc. Am. Math. Soc. 132, 885 (2004). 